\section{Kallikrein-related peptidase 4, matrix metalloproteinase 20 , and the maturation of murine and porcine enamel}

Hu Y, Hu JC-C, Smith CE, Bartlett JD, Simmer JP. Kallikrein-related peptidase 4, matrix metalloproteinase 20, and the maturation of murine and porcine enamel. Eur J Oral Sci 2011; 119 (Suppl. 1): 217-225. (C) 2011 Eur J Oral Sci

The crowns of matrix metalloproteinase 20 ( Mmp20) null mice fracture at the dentinoenamel junction (DEJ), whereas the crowns of kallikrein-related peptidase $4(K l k 4)$ null mice fracture in the deep enamel just above the DEJ. We used backscatter scanning electron microscopy to assess enamel mineralization in incisors from 9-wkold wild-type, Klk4 null, and Mmp20 null mice, and in developing pig molars. We observed a line of hypermineralization along the DEJ in developing wild-type mouse and pig teeth. This line was discernible from the early secretory stage until the enamel in the maturation stage reached a similar density. The line was apparent in Klk4 null mice, but absent in Mmp20 null mice. Enamel in the Klk4 null mice matured normally at the surface, but was progressively less mineralized with depth. Enamel in the Mmp20 null mice formed as a mineral bilayer, with neither layer looking like true enamel. The most superficial mineral layer expanded during the maturation stage and formed irregular surface nodules. A surprising finding was the observation of electron backscatter from mid-maturation wild-type ameloblasts, which we attributed to the accumulation and release of iron. We conclude that enamel breaks in the deep enamel of Klk4 null mice because of decreasing enamel maturation with depth, and at the DEJ in Mmp20 null mice because of hypomineralization at the DEJ.

\section{Yuanyuan $\mathrm{Hu}^{1}$, Jan C-C. $\mathrm{Hu}^{1}$ Charles E. Smith ${ }^{1,2}$, John D. Bartlett $^{3}$, James P. Simmer ${ }^{1}$}

${ }^{1}$ Department of Biologic and Materials Sciences, University of Michigan School of Dentistry, Ann Arbor, MI, USA; ${ }^{2}$ Facility for

Electron Microscopy Research, Department of Anatomy \& Cell Biology, and Faculty of Dentistry, McGill University, Montreal, QC Canada; ${ }^{3}$ Department of Cytokine Biology, Forsyth Institute and Department of

Developmental Biology, Harvard School of

Dental Medicine, Cambridge, MA, USA
James P. Simmer, Department of Biologic and Materials Sciences, University of Michigan Dental Research Lab, 1210 Eisenhower Place, Ann Arbor, MI 48108, USA

Telefax: +1-734-9759329

E-mail: jsimmer@umich.edu

Key words: backscatter scanning electron microscopy; energy-dispersive X-ray analysis; proteases; proteinases; teeth

Accepted for publication July 2011
Dental enamel forms in two stages. During the secretory stage, enamel mineral ribbons initiate on the surface of mineralizing dentin. Each ribbon appears to elongate by the incremental addition of amorphous calcium phosphate (1) at a mineralization front along the secretory surface of ameloblasts (2). This process requires the secretion of amelogenin $(3,4)$, ameloblastin $(5,6)$, enamelin $(7,8)$, and matrix metalloproteinase 20 (MMP20) $(9,10)$. By the end of the secretory stage, the enamel layer has achieved its final thickness, but is hypomineralized and still contains accumulated organic matrix comprised largely of enamel protein cleavage products. During the subsequent maturation stage, ameloblasts secrete kallikrein-related peptidase 4 (KLK4) $(11,12)$ and resorb the residual organic matrix. The enamel layer hardens as the crystallites formed during the secretory stage grow in width and thickness until they come into contact with adjacent crystallites and interlock (13). Thus, two secreted proteinases - MMP20 and KLK4 are necessary for proper dental enamel formation (14).

To gain a better understanding of the roles played by MMP20 and KLK4 in dental enamel formation, we used backscatter scanning electron microscopy to characterize enamel mineralization in incisors from 9-wk-old wild- type, Klk4 null, and Mmp20 null mice, and in developing pig molars. Backscattered scanning electron microscopy images can distinguish tissues that vary in their degree of mineralization because the quantity of high-energy backscattered (reflected) electrons increases as the atomic numbers of the atoms in the specimen increases. The more highly mineralized a tissue is, the more calcium (atomic number $=20$ ) there is to scatter electrons. As the mineral composition, in terms of the nature of the mineral ion constituents, remains relatively the same across the enamel layer, differences in backscattering reflect differences in mineral density or mineral content. A backscattered electron image resembles a radiograph or image generated by micro-computed tomography $(\mu \mathrm{CT})$, although $\mu \mathrm{CT}$ provides three-dimensional (3D) images while backscattered scanning electron microscopy is two dimensional (2D). When the quantity of backscattered electrons is low, the scanning electron microscopy image is black. This is generally true for soft tissues. Mature dental enamel appears white. Developing dentin, bone, and enamel show varying shades of gray, depending upon their levels of mineralization.

Using computer software, we assigned a different colour to parts of backscattered scanning electron 
microscopy images that fell within one of five ranges of gray intensity. All areas in a range were filled with the same colour, regardless of whether they were at the top or the bottom of the range. Some caution must be exercised while interpreting these images. The colourization highlights, but potentially exaggerates, differences in degrees of mineralization, so impressions gained from the colour images need to be reassessed by examining the gray-scale scanning electron microscopy images. There is also the potential for edge effects where there is contact between high- and low-reflecting surfaces, so that the edges of cracks appear to be hypermineralized, and early events at the dentino-enamel junction (DEJ), before the enamel layer is sufficiently mineralized to backscatter electrons, must be interpreted with caution.

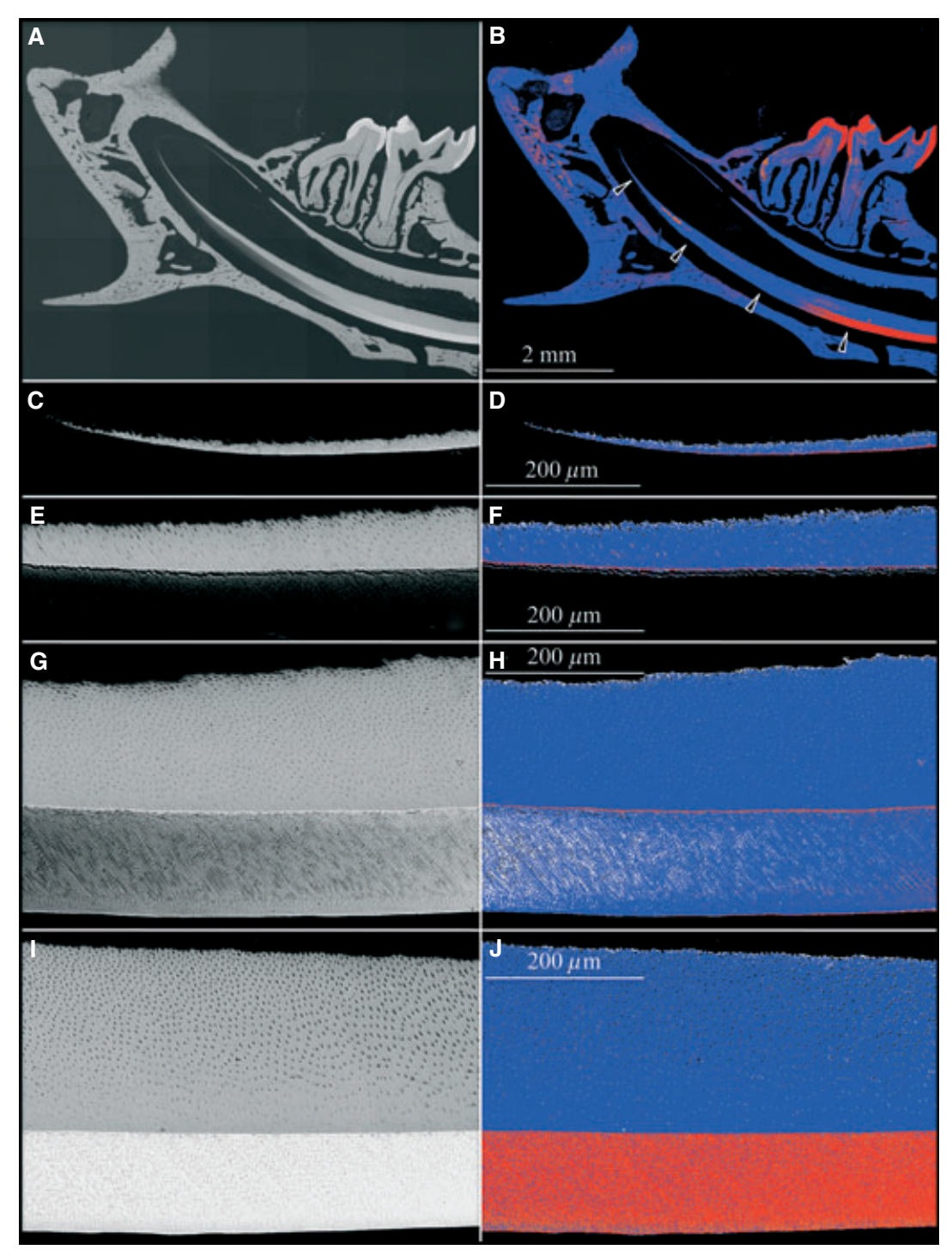

Fig. 1. Backscatter scanning electron microscopy longitudinal section of the mandible of a 9-wk-old wild-type mouse. Left column: backscatter scanning electron microscopy images. Right column: five-colour gray-scale level colour mapping of the images shown on the left, using ImageJ, with black, white, blue, orange, and red showing increasing levels of mineral density. All images are oriented so that the apical end is at the left and the incisal end is at right; the incisor dentin is above and the enamel below. (A, B) Lowmagnification mosaic of backscatter scanning electron microscopy images. Four arrows indicate the approximate positions of the subsequent high-magnification images. (C, D) Apical end of incisor. A thin white (left image) or red (right image) line is evident on the dentin surface facing the enamel, which is not sufficiently mineralized to backscatter electrons. (E, F) Early secretory stage. An artifactual separation of the dentin and enamel layers is present, causing a minor 'edge effect'. The line on the dentin side is stronger, suggesting that it is more than just an edge effect. $(\mathrm{G}, \mathrm{H})$ End of secretory stage. Mineralization in enamel appears to be greatest near the dentino-enamel junction (DEJ) and the enamel surface, with the intervening enamel being slightly less mineralized. The hypermineralized line is at the same location (at the DEJ), as noted in the more apical segments, but is more prominent and there is now no question of an edge effect. (I, J) Maturation stage. The enamel layer has increased in mineralization so that the DEJ no longer appears hypermineralized with respect to the rest of the enamel layer. 


\section{Material and methods}

\section{Tooth specimens}

All procedures involving animals were reviewed and approved by the University Animal Care Unit Committee (UACUC) at the University of Michigan. First and second mandibular and maxillary molars were obtained from 6- and 9-month-old pigs as they were processed at the Michigan State University Meat Laboratory. Mouse mandibles were dissected from 9-wk-old C57BL/6 wild-type, Klk4 null (12), and Mmp20 null (10) mice. Human bicuspids from a 14-yr-old patient were obtained after being extracted for orthodontic reasons at the University Hospital (Ann Arbor, MI, USA).

\section{Scanning electron microscopy}

Tissue processing: 9-wk-old mice were anaesthetized with isoflurane and fixed by cardiac perfusion. Blood was cleared from the vasculature using lactated Ringer's solution (30$45 \mathrm{~s}$ ) followed by $4 \%$ paraformaldehyde in PBS (135 mM $\mathrm{NaCl}, 2.7 \mathrm{mM} \mathrm{KCl}, 4.3 \mathrm{mM} \mathrm{Na} \mathrm{HPO}_{4}, 1.4 \mathrm{mM} \mathrm{Na}_{2} \mathrm{H}_{2-}$ $\mathrm{PO}_{4}$; pH 7.3) for $20 \mathrm{~min}$. The mouse mandibles were removed and stripped of soft tissue. The soft tissue was also removed from the human and pig teeth, and all were fixed by immersion in $4 \%$ paraformaldehyde overnight and washed in PBS 3x (every $0.5-1 \mathrm{~h}$ ) with one overnight wash at $4^{\circ} \mathrm{C}$.

Embedding: The pig and human teeth and the mouse mandibles were dehydrated in $30 \%, 50 \%, 70 \%, 80 \%, 90 \%$, and $100 \%$ acetone, for $30 \mathrm{~min}$ each (twice for $100 \%$ acetone), and then infiltrated with Epon (Ted Pella, Redding, CA, USA)/acetone $[1: 1$, volume by volume $(\mathrm{v} / \mathrm{v})]$ overnight. The next morning, the specimens were infiltrated with Epon/acetone $(2: 1, \mathrm{v} / \mathrm{v})$ followed by Epon/acetone $(3: 1, \mathrm{v} / \mathrm{v})$ in the afternoon. On the third day, infiltration was performed with fresh Epon/acetone $(3: 1, \mathrm{v} / \mathrm{v})$ in the morning and with $100 \%$ Epon in the afternoon. On the fourth day, the specimens were infiltrated with pure Epon in the morning, degassed under a vacuum for $4 \mathrm{~h}$, labelled, incubated at room temperature for $1 \mathrm{~h}$, and allowed to polymerize for $3 \mathrm{~d}$ at $58^{\circ} \mathrm{C}$.

Polishing: The Epon blocks containing the embedded specimens were trimmed with a coarse rotary diamond wheel on a Model 650 Low Speed Diamond Wheel Saw (South Bay Technology, San Clemente, CA, USA) and then sectioned along the plane to be examined using a fine diamond wheel. Some mouse mandibles were radially cross-sectioned to examine the continuously growing mandibular incisor at $1-\mathrm{mm}$ increments. The cut Epon blocks were placed face down in a $25-\mathrm{mm}$ SeriForm mounting cup (Struers, Ballerup, Denmark), and the plastic support was thickened by the addition of castolite AC (Woodstock, IL, USA) and polymerized at room temperature overnight. The blocks were polished with successively finer grades $(400,600$, and 800) of silicone carbide paper (South Bay Technology) followed by $16 \mathrm{~h}$ of polishing with 1.0 micro alumina abrasive on a syntronvibrating polisher. The polished block surface was cleaned by sonication and air-dried.

Imaging: The polished surface was coated in carbon and examined at $\times 50$ magnification in a Cameca SX-100 Electron Microprobe Analyzer (Madison, WI, USA) using the backscatter mode at a beam current of $15 \mathrm{kV}$ and $10 \mathrm{nA}$. Images were captured at a working distance of $9 \mathrm{~mm}$, with minor adjustments to focus. A computer-controlled stage and associated software were used to create montages of large block faces. Five-level colour mapping was performed using ImageJ (http://rsb.info.nih.gov/ij/) on TIFF images that were normalized to have the same mean gray-level intensities for mineralized bone. For pseudo colourization of the images, gray levels 1-57 were assigned as black, 58-97 as white, $98-162$ as blue, $163-192$ as orange, and $193-255$ as red. These settings were saved in a look-up table and applied to all images in the study. For the comparative backscatter scanning electron microscopy imaging, a calibration standard was not used; instead, the intensity of backscatter from the bone surrounding the tooth was used for calibration. Differences in bone density among the samples would have caused similar variations in the apparent densities of enamel and dentin in the samples being compared.

\section{Energy-dispersive X-ray analysis}

Energy-dispersive X-ray analysis (EDS) is a method for micro-analyzing the elemental constituents within a target region of a sample being characterized by scanning electron microscopy. The electron beam is focused on the target region, which ionizes elements and replaces the lost electrons with higher-energy electrons that emit X-rays in the process. By analyzing the emitted X-rays, the elemental composition of the sample can be determined. EDS was collected by the Cameca SX-100 Electron Microprobe Analyzer equipped with an energy-dispersive X-ray detector at $15.0 \mathrm{kV}$.

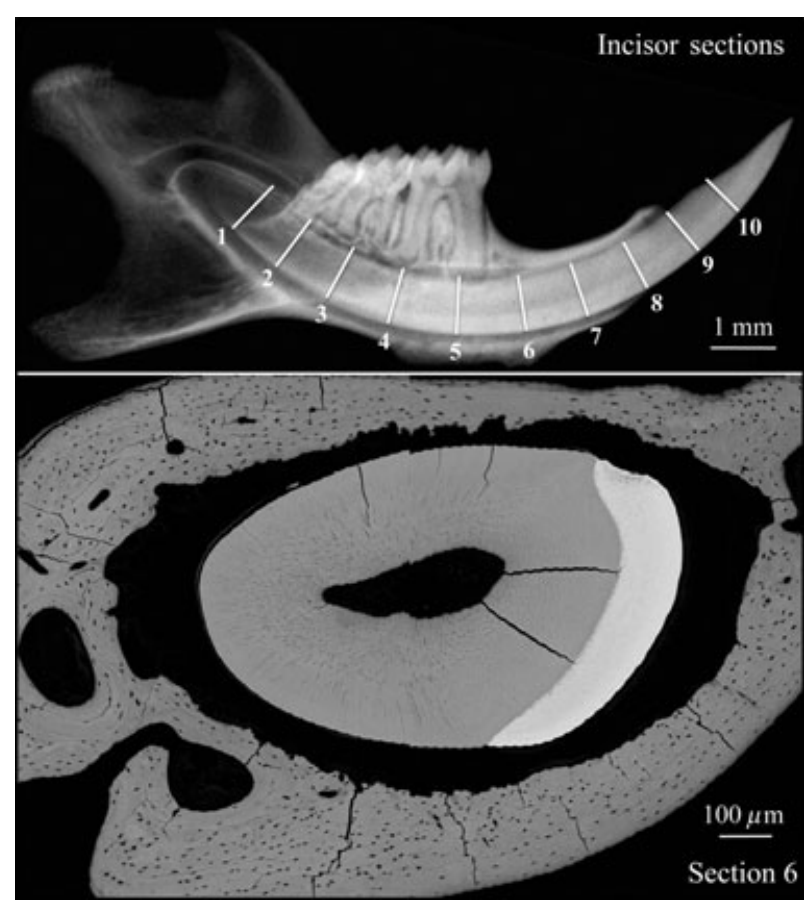

Fig. 2. Radiograph (upper image) showing the approximate positions of incisor cross-sections on a mouse mandible at $9 \mathrm{wk}$, and a backscatter scanning electron microscopy image (lower image) of a cross-section sliced at position 6. In Fig. 3 we show incisor backscatter scanning electron microscopy images that we estimate to be from sections 2, 4, and 6 shown in the upper image of this figure, although the level of precision might have varied by as much as $1 \mathrm{~mm}$. 


\section{Results}

The continuously erupting rodent incisor is a particularly useful model for studying tooth mineralization because all stages of enamel formation are displayed linearly, with early events at the apical end and late events at the incisal tip. We examined the apical half of the 9-wk mouse mandibular incisor in longitudinal sections (Fig. 1) and in cross-sections (Figs 2, 3) by scanning electron microscopy in the backscatter mode. At the
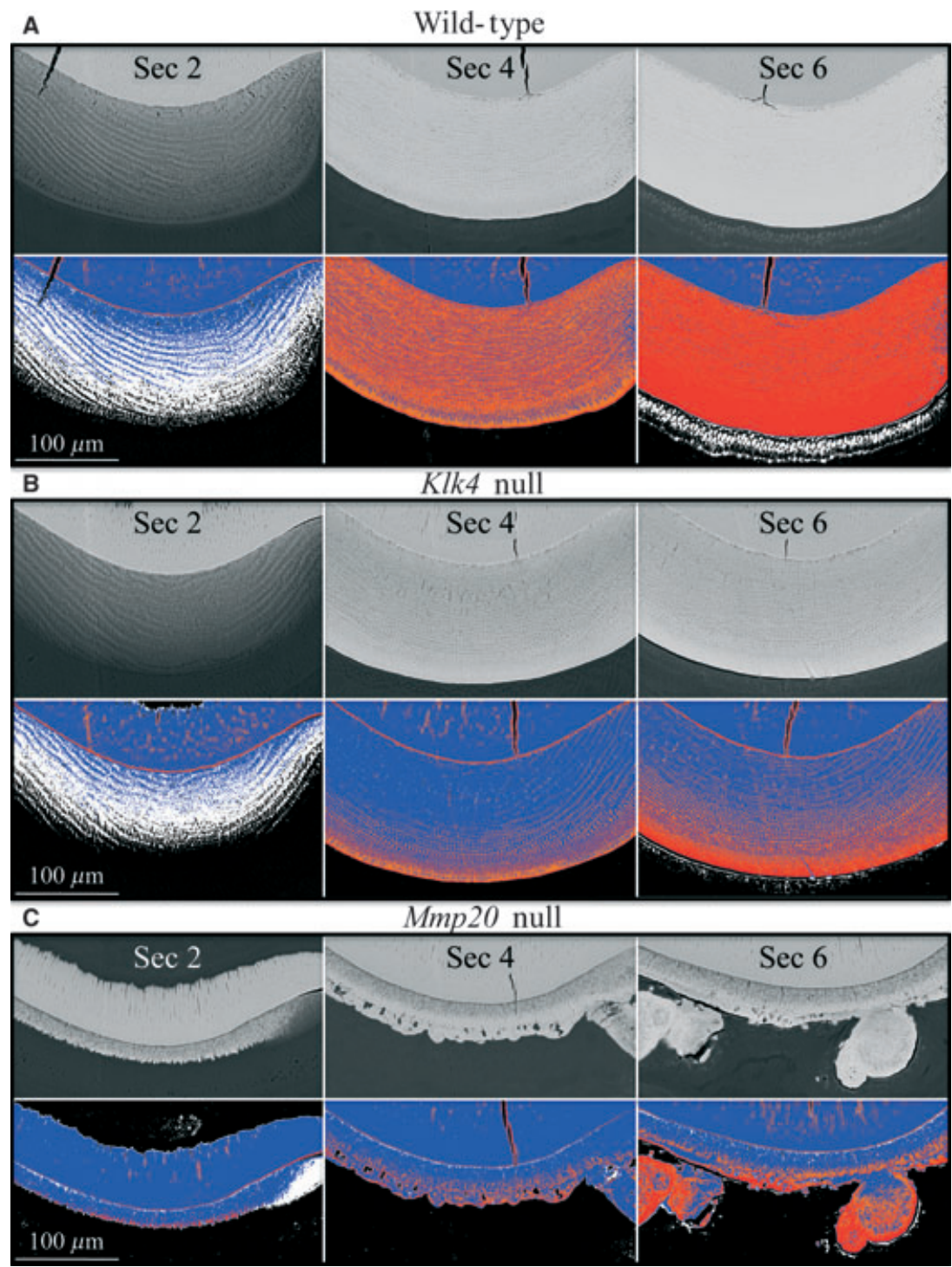

Fig. 3. Backscatter scanning electron microscopy images from 9-wk-old wild-type, kallikrein-related peptidase 4 (Klk4) null, and matrix metalloproteinase 20 ( Mmp20) null mouse incisor cross-sections. The cross-sections (from left to right) are from sectional positions 2 (late secretory), 4 (early maturation), and 6 (mid-maturation) of the mouse incisor, as shown in Fig. 2 . The row immediately below each panel of backscattered scanning electron microscopy images shows five-colour mapping of the image above, with black, white, blue, orange, and red representing increasing levels of mineralization. (A) Wild-type mouse. Note that ameloblasts produce a backscatter image as though they are mineralized. This phenomenon is associated with the accumulation and subsequent transportation of iron into the enamel. (B) Klk4 null mouse. The enamel layer in late secretory stage is comparable with that of the wild-type mouse with respect to thickness, and maturation of the enamel appears normal on the surface, but is progressively undermineralized with depth. Backscattering of electrons from mid-maturation ameloblasts is detected, but appears to be less than in wild-type mice. (C) Mmp20 null mouse. At the late secretory stage an initial mineral layer without the texture of rod/inter-rod enamel covers dentin, and no hypermineralized line at the dentino-enamel junction (DEJ) is observed. During early maturation, a second mineralized layer appears to be deposited on top of the first layer. The deeper part of the inner layer appears to mature slowly, perhaps in part because of the retention of enamel proteins. The increase in mineral density of the superficial portion of the inner layer is uneven (orange and blue mineral densities). Unlike in the formation of normal enamel where the full thickness of the enamel layer is established in the secretory stage and does not increase later, the mineral layer covering dentin in the Mmp20 null mice thickens during the maturation stage, particularly in areas where mineralized nodules form. The backscatter images were normalized to have the same mean gray-level intensity as the alveolar bone at the level of the cemento-enamel junction. 
apical end of the incisor, dentin alone generates a backscatter image, which shows increases in the intensity and the thickness of dentin in the apical-to-incisal direction (Fig. 1C,D). The intensity was greatest along a line on the dentin surface at the DEJ, although in the most apical section it was not clear if this line was caused by a higher degree of mineralization or an edge effect, as the adjacent enamel layer was not sufficiently mineralized to backscatter electrons (Fig. 1C,D). Moving incisally, the dentin layer showed a steady increase in thickness and still showed a distinctly strong signal along the DEJ, as the deepest part of the enamel layer became discernible (Fig. 1E,F). At the next level the full thickness of the enamel layer was apparent and achieved a density similar to that of the underlying bulk dentin (Fig. 1G,H). However, the highly mineralized line at the DEJ was still evident and had thickened, which is clearly not an edge effect as the adjacent dentin and enamel are both sufficiently mineralized to backscatter electrons. Eventually the entire enamel layer became highly mineralized and the line along the DEJ could no longer be distinguished (Fig. 1I,J).

We also used backscatter scanning electron microscopy to assess the relative levels of mineralization in mouse mandibular incisor cross-sections for wild-type, Klk4 null, and Mmp20 null mice (Figs 2, 3). The approximate positions of the cross-sections in the $9 \mathrm{wk}$ developing incisor are shown in Fig. 2. The highly mineralized line at the DEJ, apparent in the longitudinal sections (Fig. 1), was also evident in the secretory stage (section 2) cross-sections from the wild-type (Fig. 3A) and Klk4 null (Fig. 3B) mouse incisors, but was reduced and discontinuous in the Mmp20 null mouse incisor (Fig. 3C). Early maturation enamel (section 4) in the incisor from the wild-type mouse was highly mineralized, but speckled with areas of reduced mineral density. The spots corresponded to places where the enamel rods were cut in cross-section and part of the electron beam passed through inter-rod enamel, which is less mineralized than rod enamel at this stage. Secretory-stage enamel (section 2) in the Klk4 null mouse looked similar to that of the wild-type mouse. The Klk4 null mouse maturation-stage sections looked similar to those of the wild-type at the surface only, with the enamel showing progressively less mineralization with depth. The maturation deficit in the Klk4 null mice was predominantly manifested as an inability to mineralize the deeper enamel.

The enamel from the Mmp20 null mouse displayed two well-delineated layers, neither of which resembled wildtype enamel (Fig. 3C). The layer covering dentin was deposited during the secretory stage. It appeared to be fairly uniform in thickness $(25-30 \mu \mathrm{m})$, but did not have
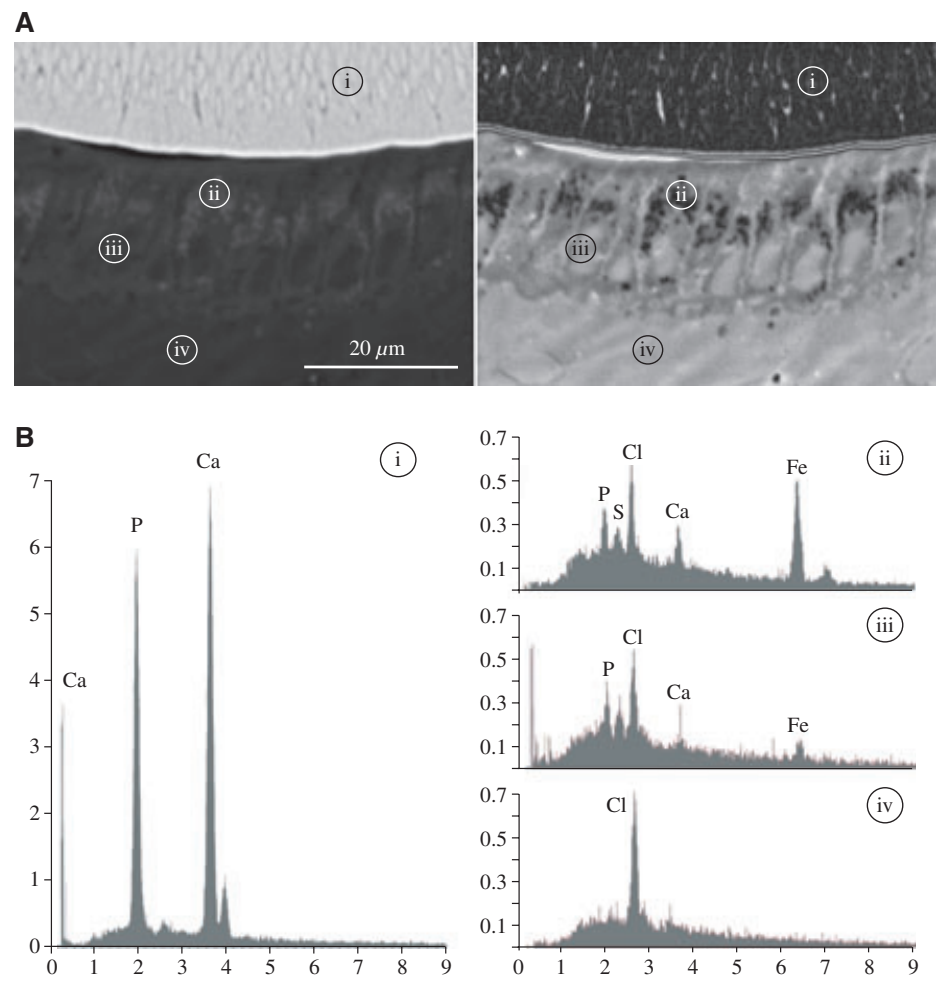

Fig. 4. Backscatter scanning electron microscopy and energy-dispersive X-ray analysis (EDS). (A) Backscatter scanning electron microscopy of the incisor cross-section at the level of section 5 (Fig. 2) localizing the four sites where elemental analyses were performed. Left: backscatter scanning electron microscopy image. Right: backscatter scanning electron microscopy image that was sharpened and inverted using ImageJ to better show the backscatter from the ameloblast layer. (B) EDS spectra from the four areas localized in panel A: i, enamel layer; ii, ameloblast area with positive backscatter; iii, ameloblast nucleus without positive backscatter; and iv, papillary layer. Note the presence of iron ( $\mathrm{Fe}$, atomic number 26$)$ in area ii and that the levels of calcium $(\mathrm{Ca})$ and phosphorus (P) are also high. $\mathrm{Cl}$, chlorine; $\mathrm{S}$, sulphur. The $x$-axis numbers represent kiloelectron volts $(\mathrm{keV})$, and the $y$-axis numbers represent counts per second per electron volt $(\mathrm{CPS} / \mathrm{eV})$. 
a speckled pattern characteristic of rod/inter-rod organization, and did not progressively mineralize during the maturation stage. The outer layer was irregular in thickness and appeared to form largely in the maturation stage. A major feature of this second layer was mineralized surface nodules that varied greatly in their dimensions. The outer layer became progressively more mineralized during the maturation stage, but not in all areas.

A surprising finding in the wild-type incisors was discernible backscatter from maturation-stage ameloblasts. The EDS results suggested that this signal was caused by the accumulation and subsequent transport of iron, which gives the mouse incisors their yellowish-brown colour (Fig 4). Although iron was the main element present at the site of soft-tissue backscatter, calcium and phosphate were also detected, and further studies are required to understand this phenomenon. Discernible backscatter from the maturation ameloblast layer was reduced in the Klk4 null mouse and at the lowest level in the Mmp20 null mouse.

We examined a developing (unerupted) second molar from 9-month-old pigs, which had completed the secretory stage but had a crown at varying stages of maturation (Fig. 5A,B): the enamel was progressively less mature from the direction of the cusp tip to the cervical margin. As in the mouse incisors, a well-demarcated, highly mineralized layer was observed at the DEJ, which could no longer be distinguished after the overlying enamel reached a similar level of mineralization (Fig. 5 C,D). In the porcine first molar at 6 months, which is more advanced developmentally and already erupted, the enamel was fully mature and highly mineralized in all areas (Fig. 6, 6C). Maturation of the porcine enamel layer was more advanced with depth and occurred latest at the enamel surface. The mineral density of the enamel layer in the erupted first molar of the 9-wk-old mouse was not the same in all areas, but seemed to be highest near the DEJ and enamel surface and sometimes less mineralized in the middle (Fig. 6A). We examined a human bicuspid from a 14-yr-old patient, but mineralization was already complete in all areas of the crown (Fig. 6D). Thus, a highly mineralized layer at the DEJ is a temporary feature that initially appears on the dentin surface during the secretory stage and is apparent until the adjacent enamel layer matures to a similar level of mineralization.

\section{Discussion}

In this study we used backscatter scanning electron microscopy to examine the progressive mineralization of developing teeth. An important finding was that we were able to trace the origins of the thin, highly mineralized layer along the DEJ to the secretory stage of development and confirmed earlier observations that the highly mineralized line at the DEJ is present in wild-type and Klk4 null mice, but not in the Mmp20 null mice (15). We rejected the hypothesis that this highly mineralized layer is an artifact (edge effect) because it is greatly reduced in

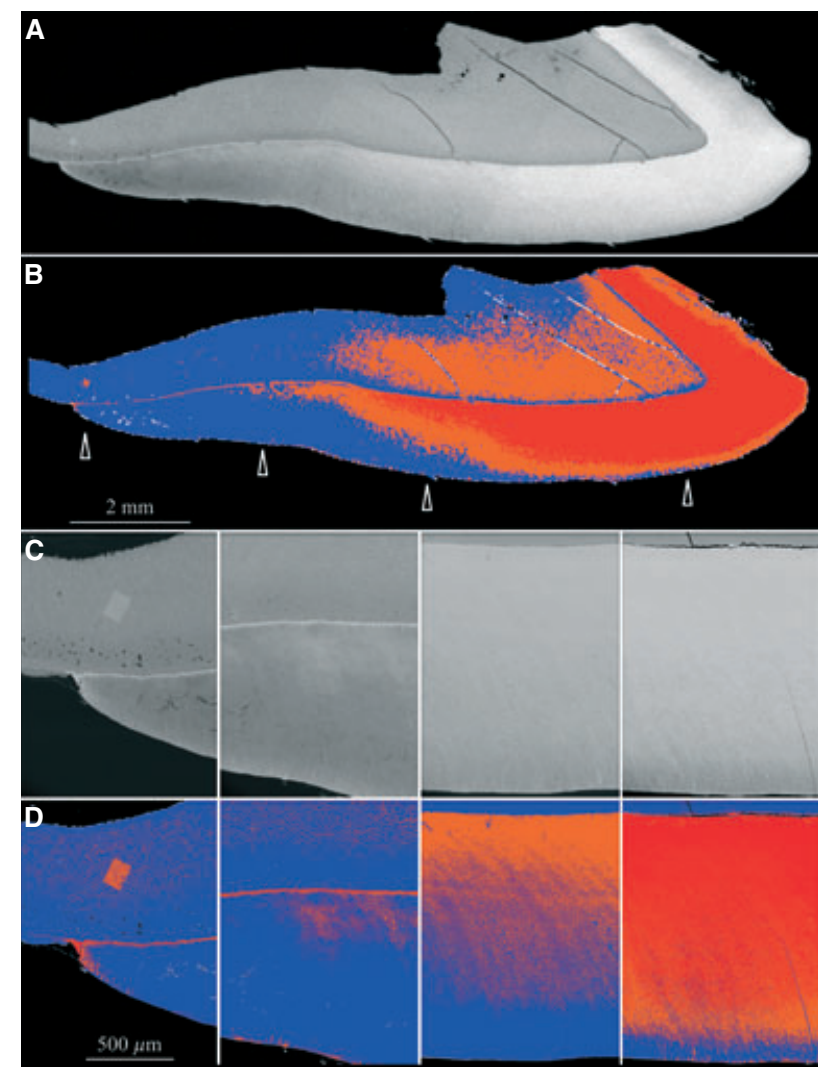

Fig. 5. Backscatter scanning electron microscopy images of a single second molar cusp from a 9-month-old pig. All images are oriented so that cervical is left and cuspal is right. Dentin is above and enamel is below. Beneath each scanning electron microscopy image are five levels of intensity colour mappings, with black, white, blue, orange, and red representing increasing levels of mineralization. (A, B) Low-magnification mosaic of backscatter scanning electron microscopy images. Four arrows indicate the approximate positions of the subsequent highmagnification images. Minor discontinuities in the hypermineralized line are caused by imperfections in the computer assembly of the mosaic. (C, D) High-magnification backscatter scanning electron microscopy images. Near the cervical region (left) there is a hypermineralized line evident at the dentinoenamel junction (DEJ). In the pig this line is thick and extends into the enamel layer. The enamel layer first matures near the DEJ and progresses from there towards the enamel surface. The high-density rectangle in dentin near the cervical margin was caused by beam damage. The backscatter images were normalized to have the same mean gray-level intensity as that of the alveolar bone in the 9-wk-old wild-type mouse (Fig. 3).

Mmp20 null mice, which produce normal dentin, and would be expected to show the same edge effect as the other mice. In fact, the faint interrupted line in the Mmp20 null mice appears to show how small the edge effect is at the outer dentin surface. Additional evidence that the highly mineralized line along the DEJ is more than an edge effect is the observation that it does not disappear when the enamel layer achieves a mineral density similar to that of dentin (when there is no 'edge'), but instead becomes thicker and more obvious.

A highly mineralized layer at or above the DEJ in developing porcine teeth has long been recognized (16). 


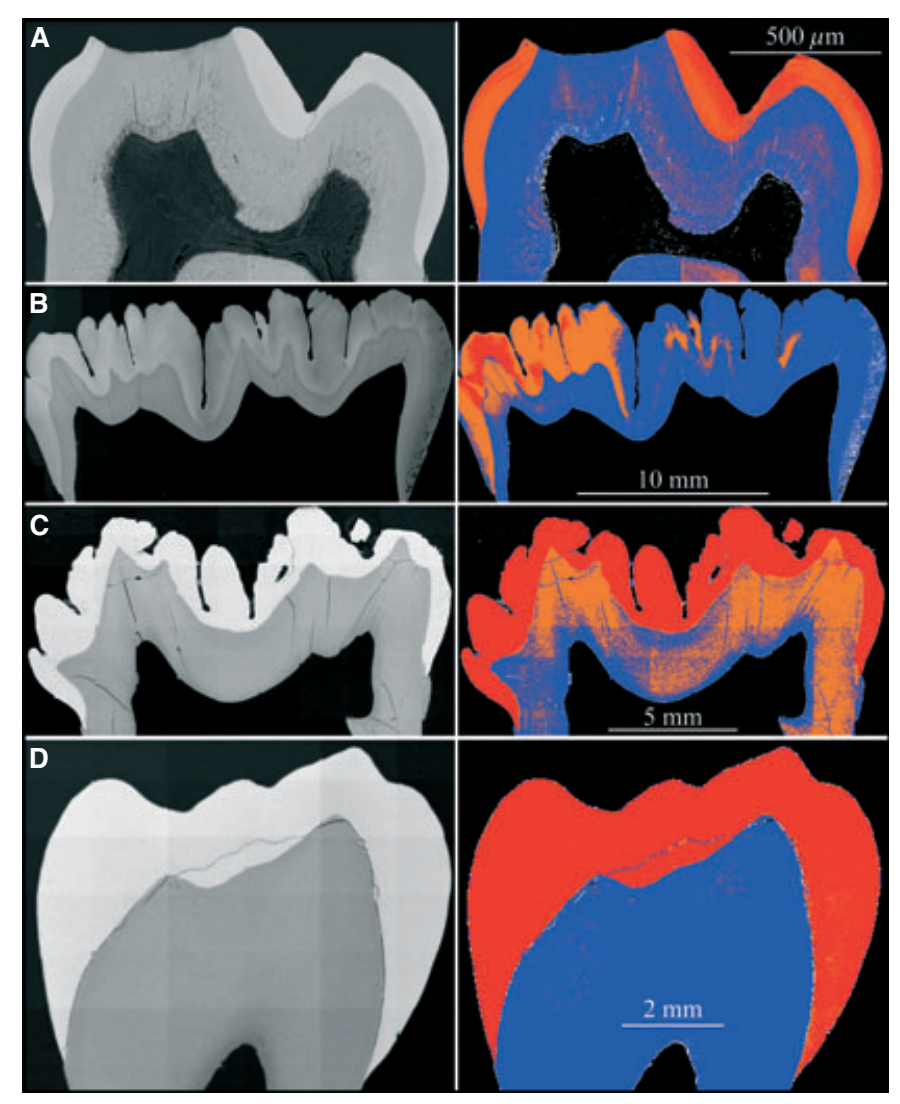

Fig. 6. Backscatter scanning electron microscopy images of mouse, pig, and human molars. (A) First molar from 9-wk-old mouse. Enamel maturation is too advanced to detect a hypermineralized line at the dentino-enamel junction (DEJ). The maturation-stage crown is slightly more mineralized at the surface compared with the deeper regions of the enamel near the dentin. (B) Second molar from a 6-month-old pig. This unerupted molar has just finished the crown-formation stage and is starting to form roots. One side has a more advanced level of mineralization within the enamel layer. A hypermineralized line is not evident in the colour image but can be distinguished covering dentin near the cervical margin on the left. (C) First molar from a 6-month-old pig. This erupted molar is mature beyond the point where a hypermineralized line along the DEJ can be observed. (D) Premolar from a 14-yr-old human. The enamel layer is fully mature. The backscatter images were normalized to have the same mean gray-level intensity as that of the alveolar bone in the 9-wk-old wild-type mouse (Fig. 3).

Previously, it was proposed that this layer hypermineralizes because odontoblasts secrete KLK 4 through their processes at the DEJ (17). However, in Klk4 null mice, the coding region for KLK4 was replaced with a LacZ reporter, and histochemical analyses clearly demonstrated that KLK4 is not normally expressed by mouse secretory-stage ameloblasts or by the odontoblasts beneath them (18). These data strongly suggested that KLK4 is not present during formation of the DEJ in mice. It is also unlikely that Klk4 expression patterns vary significantly between pigs and mice. Accumulated amelogenins $(19-21)$ and ameloblastins $(22,23)$ in porcine secretory-stage enamel are cleaved at the same sites that are generated by MMP20 in vitro, so KLK4 does not make its presence felt sufficiently in terms of its contribution to accumulated cleavage products to support the argument that KLK4 is active in porcine secretory-stage enamel. We conclude that MMP20 is critically important for the formation of a normal DEJ (24), while KLK4 is not. This conclusion is also supported by the observation that enamel fractures at the
DEJ in Mmp20 null mice, but in the enamel layer above the DEJ in the Klk4 null mice $(12,18)$. The apparent reason for Klk4 null enamel fracturing deep within the enamel layer is caused by the progressive failure of enamel maturation with depth, so that the weakest part is just above the DEJ. However, it is not clear why the enamel is able to mature at the surface without KLK4. We suspect that continued expression of MMP20 and endocytosis by ameloblasts combine to remove proteins from the surface enamel, but that KLK4 may be necessary to break up aggregates of accumulated enamel protein cleavage products so that they can return to the ameloblast for endocytosis.

The continued characterization of Mmp20 null mice increases appreciation for its central role in DEJ and enamel formation. MMP20-catalyzed cleavages are critical for development of a highly mineralized dentin surface during early crown formation and for subsequent organization of the enamel layer. The mineral bilayer that forms over dentin in Mmp20 null mice bears little resemblance to true enamel and is capped by mineralized 
nodules. Our current interpretation of events in the Mmp20 null mouse is that during the secretory stage a 25-30 $\mu \mathrm{m}$ defective mineralized layer is deposited on top of the DEJ and contains abundant uncleaved enamel proteins. During the maturation stage, ions that normally contribute to the maturation of crystallites deposited during the secretory stage cannot penetrate the inner mineral layer and instead precipitate as a second layer on top of the first. In wild-type mice, Mmp20 catalyzed cleavages are necessary to hypermineralize the DEJ, maintain the mineralization front where enamel crystals elongate, cleave enamel proteins to promote their reabsorption by ameloblasts, and to sustain the ameloblast Tomes' process, potentially through a mechanism involving the cleavage of E-cadherin (25).

The disturbances of enamel formation observed in the various knockout mice affect ameloblasts more than might have been predicted by the classical view that ameloblasts create a well-defined extracellular space conducive for mineralization and secrete enamel proteins to project control over that mineralization from a distance. The process of enamel formation requires the formation of a mineralization front along the ameloblast cell membrane to generate mineral ribbons. Sustaining the mineralization front requires the secretion, processing, selective accumulation, and removal of organic matrix, and also requires continuous cell-matrix interactions to adjust for the constantly changing requirements of mineral ribbon deposition and elongation. Enamel formation requires an ameloblast Tomes' process to establish rod/inter-rod organization and the coordinated movement of ameloblasts to produce the decussating rod architecture and to thicken the enamel layer. Without proper enamel formation, ameloblasts cannot sustain their phenotype, so the process of enamel formation requires proper differentiation of ameloblasts and then the process becomes more physiological (responding to matrix feedback mechanisms) than developmental (responding to epithelial-mesenchymal interactions). Successful production of initial enamel is required to provide extracellular stimuli necessary to maintain healthy ameloblasts.

Acknowledgements - We thank Mr Tom Forton, Manager of the Michigan State University Meat Laboratory, and members of the Michigan State University Department of Animal Science for their kind assistance in obtaining fresh developing molars from pigs slaughtered at their facility. We thank Dr Yasuo Yamakoshi of the University of Michigan for the extraction and initial processing of the pig molars. We thank Dr Brent Ward and Tammie Schroka of University Hospital and Laney Mai of the University of Michigan School of Dentistry for the extraction and initial processing of the human premolars. We thank Carl Henderson of the Electron Microbeam Analysis Laboratory (EMAL) in the Department of Geological Sciences at the University of Michigan for his assistance in the backscatter imaging. This investigation was supported by USPHS Research Grants DE019775 and DE016276 from the National Institute of Dental and Craniofacial Research, National Institutes of Health, Bethesda, MD 29892.

Conflicts of interest - All authors declare that there are no competing interests.

\section{References}

1. Beniash E, Metzler RA, Lam RS, Gilbert PU. Transient amorphous calcium phosphate in forming enamel. $J$ Struct Biol 2009; 166: 133-143.

2. Simmer JP, Papagerakis P, Smith CE, Fisher DC, Rountrey AN, ZHENG L, Hu JC. Regulation of dental enamel shape and hardness. J Dent Res 2010; 89: 1024-1038.

3. Snead Ml, Lau EC, Zeichner-David M, Fincham AG, Woo SL, SLAVkIN HC. DNA sequence for cloned cDNA for murine amelogenin reveal the amino acid sequence for enamel-specific protein. Biochem Biophys Res Commun 1985; 129: 812-818.

4. Gibson CW, Yuan ZA, Hall B, Longenecker G, Chen E, Thyagarajan T, Sreenath T, Wright JT, Decker S, Piddington R, Harrison G, Kulkarni AB. Amelogenin-deficient mice display an amelogenesis imperfecta phenotype. $J$ Biol Chem 2001; 276: 31871-31875.

5. Krebsbach PH, Lee SK, Matsuki Y, Kozak CA, Yamada K, YAMADA Y. Full-length sequence, localization, and chromosomal mapping of ameloblastin: a novel tooth-specific gene. $J$ Biol Chem 1996; 271: 4431-4435.

6. Fukumoto S, Kiba T, Hall B, Iehara N, Nakamura T, Longenecker G, Krebsbach PH, Nanci A, Kulkarni AB, YAMADA Y. Ameloblastin is a cell adhesion molecule required for maintaining the differentiation state of ameloblasts. J Cell Biol 2004; 167: 973-983.

7. Hu CC, Fukae M, Uchida T, Qian Q, Zhang CH, Ryu OH, Tanabe T, Yamakoshi Y, Murakami C, Dohi N, Shimizu M, SIMMER JP. Cloning and characterization of porcine enamelin mRNAs. J Dent Res 1997; 76: 1720-1729.

8. Hu JC, Hu Y, Smith CE, McKee MD, Wright JT, Yamakoshi Y, Papagerakis P, Hunter GK, Feng JQ, YAMAKoshi F, Simmer JP. Enamel defects and ameloblastspecific expression in Enam knock-out/lacz knock-in mice. J Biol Chem 2008; 283: 10858-10871.

9. Bartlett JD, Simmer JP, Xue J, Margolis HC, Moreno EC. Molecular cloning and mRNA tissue distribution of a novel matrix metalloproteinase isolated from porcine enamel organ. Gene 1996; 183: 123-128.

10. Caterina JJ, Skobe Z, Shi J, Ding Y, Simmer JP, Birkedal-Hansen H, BARTLETT JD. Enamelysin (matrix metalloproteinase 20)-deficient mice display an amelogenesis imperfecta phenotype. J Biol Chem 2002; 277: 49598-49604.

11. Simmer JP, Fukae M, Tanabe T, Yamakoshi Y, Uchida T, Xue J, Margolis HC, Shimizu M, DeHart BC, Hu CC, Bartlett JD. Purification, characterization, and cloning of enamel matrix serine proteinase 1. J Dent Res 1998; 77: 377-386.

12. Simmer JP, Hu Y, Lertlam R, Yamakoshi Y, Hu JC. Hypomaturation enamel defects in Klk4 knockout/LacZ knockin mice. J Biol Chem 2009; 284: 19110-19121.

13. Sмiтн CE. Cellular and chemical events during enamel maturation. Crit Rev Oral Biol Med 1998; 9: 128-161.

14. Lu Y, Papagerakis P, Yamakoshi Y, Hu JC, Bartlett JD, SiMMER JP. Functions of KLK4 and MMP-20 in dental enamel formation. Biol Chem 2008; 389: 695-700.

15. Smith Ce, Richardson AS, Hu Y, Bartlett JD, Hu JC-C, SiMmER JP. Effects of loss of function of kallikrein 4 on mineralization of enamel: comparison to mice lacking matrix metalloproteinase 20. J Biol Chem 2011; 286: 18149-18160.

16. Suga S. Comparative histology of the progressive mineralization pattern of developing enamel. In: Suga S, ed. Mechanisms of Tooth Enamel Formation. Tokyo: Quintessence Publishing Co Inc., 1983; 167-203.

17. Fukae M, Tanabe T, Nagano T, Ando H, Yamakoshi Y, Yamada M, Simmer JP, Oida S. Odontoblasts enhance the maturation of enamel crystals by secreting EMSP1 at the enamel-dentin junction. $J$ Dent Res 2002; 81: 668-672.

18. Simmer J, Hu Y, Richardson A, Bartlett J, Hu JC-C. Why does enamel in Klk4 null mice break above the dentino-enamel junction? Cells Tissues Organs 2011; 194: 211-215.

19. Ryu OH, Fincham AG, Hu CC, Zhang C, Qian Q, Bartlett JD, Simmer JP. Characterization of recombinant pig enamelysin activity and cleavage of recombinant pig and mouse amelogenins. J Dent Res 1999; 78: 743-750. 
20. Nagano T, Kakegawa A, Yamakoshi Y, Tsuchiya S, Hu JC, Gomi K, Arai T, Bartlett JD, Simmer JP. Mmp-20 and Klk4 cleavage site preferences for amelogenin sequences. $J$ Dent Res 2009; 88: 823-828.

21. Ryu O, Hu JC, Yamakoshi Y, Villemain Jl, Cao X, Zhang C, Bartlett JD, Simmer JP. Porcine kallikrein-4 activation, glycosylation, activity, and expression in prokaryotic and eukaryotic hosts. Eur J Oral Sci 2002; 110: 358-365.

22. Iwata T, Yamakoshi Y, Hu JC, Ishikawa I, Bartlett JD, Krebsbach PH, Simmer JP. Processing of ameloblastin by MMP-20. J Dent Res 2007; 86: 153-157.
23. Chun YH, Yamakoshi Y, Yamakoshi F, Fukae M, Hu JC, BartLETt JD, Simmer JP. Cleavage site specificity of MMP-20 for secretory-stage ameloblastin. J Dent Res 2010; 89: 785-790.

24. Beniash E, Skobe Z, Bartlett JD. Formation of the dentinoenamel interface in enamelysin (MMP-20)-deficient mouse incisors. Eur J Oral Sci 2006; 114(Suppl 1): 24-29.

25. Bartlett JD, Yamakoshi Y, Simmer JP, Nanci A, Smith CE. MMP20 cleaves E-cadherin and influences ameloblast development. Cells Tissues Organs 2011; 194: 222-226. 